\title{
USO DE HERRAMIENTAS DE CALIDAD: CASO CARROCERIAS
}

\author{
Use of quality tools: bodyworks case
}

\author{
Mario Fernando Navarrete Fonseca ${ }^{1}$ \\ Fabian Xavier Martínez Ortiz ${ }^{2}$
}

\section{Resumen}

En el Ecuador, las empresas carroceras nacionales han tenido un decrecimiento económico por el ingreso al mercado de empresas que compiten con productos extranjeros como carrocerías acabadas. Esto les obliga a gestionar adecuadamente sus herramientas de calidad para ser competitivas. El objetivo es analizar diversas herramientas de calidad y su relación con las características productivas y organizacionales del sector carrocero de la provincia de Tungurahua. La población considerada fue de 112 personas (dos por cada una de las 56 empresas registradas). La técnica para la recolección de datos fue la encuesta, cuyo instrumento (cuestionario) está constituido por 23 ítems. Los resultados demuestran que la herramienta de calidad más utilizada en el sector es el diagrama de flujo. Esta herramienta permite realizar en forma gráfica las actividades que se deben desempeñar. Por esta razón, es conveniente proponer la elaboración de una metodología basada en el diagrama de flujo como herramienta de calidad para las empresas.

\section{Palabras clave}

Metalmecánica, carrocerías, herramientas de calidad, diagrama de afinidad, de interrelación y de flujo.

\begin{abstract}
In Ecuador, bodywork companies have had an economic decrease due to the entry into the national market of companies competing with foreign products like finished car bodies. For this reason, it is necessary to properly handle quality tools to be competitive. The objective of the research was to analyze different quality tools and their relationship with the productive and organizational characteristics of the bodywork sector of the province of Tungurahua. For the development of the study, the used methods were: theoretical and empirical, and quantitative. The design was approached from an exploratory, descriptive and correlational point of view. The population considered for the study was 112 people ( 2 per registered company). According to data from the CANFAC (Cámara Nacional de Fabricantes de Carrocerías) in Tungurahua there are 56 registered companies engaged in this activity. The technique for data collection was surveys, which instrument considered consisted of a questionnaire made of 23 items. The results demonstrate that the most used quality tool in the bodywork sector is the flowchart. This tool allows them to carry out in a graphic way the activities that must be carried out. For this reason, it is convenient to propose the development of a methodology based on Flowcharts as a quality tool for these companies.
\end{abstract}

\section{Key words}

Metalworking, bodyworks, quality tools, affinity, interrelation and flow diagram.

\footnotetext{
${ }^{1}$ Universidad Técnica de Cotopaxi (Ecuador). Correo electrónico: mario.navarrete4890@utc.edu.ec.

${ }^{2}$ Universidad Técnica de Cotopaxi (Ecuador). Correo electrónico: fabian.martinez@utc.edu.ec
} 


\section{Introducción}

El mundo está cambiando a pasos gigantescos, por ende, la gestión de la calidad es un tema de estudio esencial alrededor de las empresas carroceras en América Latina. La industria metalmecánica apoya con más del $16 \%$ del PIB, lo cual representa alrededor de 4 millones de empleo de forma directa y $19,7 \%$ indirectas lo cual constituye una oportunidad de crecimiento en la región. En la revista de metalmecánica proporciona un listado de países de la participación del mercado por esta actividad económica, en Argentina represento 17.0\% del valor bruto de la producción del 2013; en Brasil el $27.0 \%$ del valor agregado manufacturado en 2012; y para Colombia significó $10.4 \%$ del valor agregado en el sector manufacturero en 2012; y en México fue 31.0\% del valor agregado manufacturero en 2012, de acuerdo con datos de la Asociación Latinoamericana del Acero (Heras, Marimon y Casadesús, 2009).

La gestión de la calidad ha sido ampliamente analizada desde el punto de vista académico. De esta forma, se ha llegado a una clasificación, ya clásica, de cuatro etapas progresivas, tanto desde el punto de vista histórico, como de concepción de dicha gestión. Dichas etapas, por lo general, han sido denominadas de la forma siguiente: inspección de la calidad, control de calidad, aseguramiento de la calidad y gestión de la calidad total. Además, desde hace ya unos años son diversos los autores que afirman que nos aproximamos a una quinta era o fase de la gestión de la calidad.

Denominaron a esta quinta etapa como el "más allá de la dirección estratégica de la calidad", a fin de definir luego cuáles eran sus criterios esenciales. Con posterioridad analizaron la literatura especializada sobre esta quinta era y afirmaron que se ha consolidado la expresión gestión de la calidad estratégica, para esta fase en la que se «funden» los principios de la GCT y de la dirección estratégica. Lo que parece claro es que, como señalan, en la práctica empresarial el alcance de cada una de estas fases de la gestión de la calidad ha sido ciertamente heterogéneo, y así lo han sido también las herramientas adoptadas en cada una de las etapas o modalidades de gestión de la calidad definidas. En la literatura, sobre todo en la de carácter profesional, las herramientas concretas relacionadas con la gestión de la calidad han sido muy estudiadas (Heras, Marimon, y Casadesús, 2009).

\section{Situación actual}

En el caso específico, Ecuador es conocido por la exportación de productos como: banano, cacao, hortalizas entre otros. Todos los sectores económicos en la actualidad buscan la generación y aplicación. La metalmecánica es el sector con mayor incidencia en cuanto a la importación de materia prima, producción, postproducción y exportación. Estos insumos son esenciales para mejorar el sector de la fabricación de buses o diferentes medios de transporte.

En todo el país se producen 127 carrocerías al mes, lo que suma 1.524 unidades al año. De este total, el 65\% sale de empresas de Tungurahua. Según un estudio de la CANFAC, en todo el país, cada año se renuevan cerca de 2.200 unidades de transporte de pasajeros. Se estima que, al menos, el 90\% del nuevo parque automotor tiene carrocerías ensambladas en talleres nacionales. La calidad total es un factor predominante para el desarrollo de las empresas; en la provincia de Tungurahua, concretamente en el sector carrocero (CANFAC), se agrupan 54 empresas que producen un estimado de 195 carrocerías mensuales, esto conlleva a la generación de competencias y la búsqueda de herramientas administrativas que permitan mejorar significativamente los procesos de calidad en cada empresa carrocera (Bombón, Vayas, Villacís y Gutierrez , 2017).

Es así que el 65\% de la producción nacional de carrocerías se centralizan en Tungurahua, estableciéndose como un sector productivo importante. El crecimiento exponencial de acuerdo a datos del Ministerio Coordinador de la Producción, Empleo y Competitividad indica que en 1990 existían 27 empresas en el sector carrocero del país y para el año 2005 se evidencia 180 empresas (Fiallos, 2014).

En el Censo Nacional Económico del 2010 se detectó 183 empresas carroceras a nivel nacional, 32 localizadas en la ciudad de Ambato (INEC, 2010). De esta manera se ha logrado estimar la dinámica económica de varios sectores, como son el hierro y acero, que tienen una participación del $34.5 \%$, en relación a la materia prima como la pintura y vidrio, con un $7.7 \%$, clavos, pernos y tornillos, $4.2 \%$ de aluminio y un $6.3 \%$, según Fiallos, (2014). Es así que los nuevos requerimientos tecnológicos y de producción han llevado a las carrocerías a tomar nuevos estándares de calidad y certificación de sus procesos. De acuerdo a CANFAC (2015), el 24\% de las empresas tienen certificación de calidad ISO 9001, el $7 \%$ en proceso de obtención de calidad y el $69 \%$ aún no incurren en este territorio. La investigación contó con las variables: herramientas de calidad diagrama de afinidad, 
de interrelaciones y de flujo. El objetivo de estudio fue analizar diversas herramientas de calidad y su relación con las características productivas y organizacionales del sector carrocero de Tungurahua.

Además, se propuso dos hipótesis: (Ho) Nula: "Las herramientas de calidad no se relacionan con las características económicas del entorno carrocero de la Provincia de Tungurahua" y (Ha) Alterna: "Las herramientas de calidad se relacionan con las características económicas del entorno carrocero de la Provincia de Tungurahua que posterior fueron comprobadas.

\section{Consideraciones teóricas}

\section{Herramientas de calidad}

El Control de Calidad tuvo su origen en la producción industrial masiva de principios del siglo XX. El desarrollo de los métodos de producción en cadena planteó el primer problema de calidad, en cuanto a que ésta estaba ligada a la conformidad con las especificaciones de los productos y sus componentes: a una más alta conformidad (calidad), correspondería un número menor de desechos y reprocesos, con lo que el coste del proceso productivo, y del producto, se reduciría. Surgen entonces los primeros procedimientos de control de calidad. La función de calidad, bajo esta óptica clásica, se limita a la realización de una serie de observaciones que tienen como objetivo la verificación de la concordancia de los diferentes dispositivos y componentes a su especificación, previamente establecida. Los resultados de las observaciones permitirían separar el producto aceptable del no aceptable mediante la inspección final del producto ya terminado (Talavera, 2013).

En Estados Unidos, el liderazgo de Taylor y su "dirección científica" supuso la separación entre la planificación del trabajo y su ejecución: la planificación era realizada por distintos especialistas mientras que los capataces y operarios ejecutaban la planificación hecha por aquellos. Desde el punto de vista del control de calidad, también se segregó la producción de la inspección. Se crearon entonces departamentos de inspección, llamados de "Control de Calidad" o de "Aseguramiento de la Calidad". $\mathrm{Su}$ finalidad era separar los productos buenos de los defectuosos de forma que éstos no llegaran al cliente (Talavera, 2013).

Si bien el aumento de la productividad fue evidente, se fomentó la idea de que la calidad era materia de los departamentos especializados en la inspección de la calidad. El concepto subyacente de gestión de la calidad era que cada departamento funcional entregaba su producto al siguiente; $y$, finalmente, el departamento de calidad separaba la producción correcta de la incorrecta (Talavera, 2013).

Dada la importancia de la calidad y teniendo en cuenta que la empresa exportadora de prendas terceriza (outsourcing en inglés) todos sus procesos, se plantea un procedimiento de calidad de uso sencillo para ayudar a las MYPES de metalmecánica a mejorar su calidad y por ende sus procesos; teniendo en cuenta que estas carecen de personal técnico de control de calidad. Además, propone elaborar una organización que guíe la mejora continua según el ciclo de Shewhart (también conocido como ciclo de Deming), que es un procedimiento muy valioso para buscar una mejora, además asevera que en cualquier paso se puede necesitar metodología estadística para ir más rápido (Gándara, 2014).

A ello se explica que la palabra Kaizen que es mejoramiento; en el lugar de trabajo tiene la connotación de mejoramiento continuo que involucra a todos y está orientada al proceso. Imai, indica que el "ciclo de Deming" o también llamado Ciclo PHRA (planificar, hacer, revisar, actuar), es una herramienta muy importante para la calidad y el mejoramiento continuo (Pérez, 2017). Un estudio realizado en España revela que las herramientas de calidad promueven la generación de competitividad en las empresas, debido que contribuye en el desarrollo de políticas de desarrollo institucional en las diferentes áreas y secciones de la empresa. La única forma de generar competitividad es proponiendo medidas correctivas en los procesos operativos y administrativos que ligado a las actitudes y habilidades de la gerencia y departamentos (Molina, 2015).

Las herramientas de calidad son metodologías que permiten gestionar la calidad de una organización, para que sean eficaces y eficientes cuando se pueda identificar un problema. Por tanto, es muy importante identificar claramente el problema para poder determinar la herramienta de calidad más eficaz (Diagrama de Pareto, Ishikawa, Gantt, Deming, etc.) por los equipos de trabajo (Gándara, 2014).

\section{Diagrama de afinidad}

Este método permitió el apuntalamiento de equipos de trabajo en las empresas japonesas de las décadas 70 y 80 que, gracias al trabajo en equipo, se consolidaron en el mercado mundial, tal es el caso de la Toyota. Esta técnica 
permite en una misma sesión el aporte individual, en grupos pequeños y en asamblea concretar de manera democrática afinidades (Viega, Quintero y Romero, 2017).

Conocido también como método K-J, "metaplan o story board", es una herramienta de trabajo en grupo cuyo objetivo consiste en obtener gran cantidad de datos en forma de ideas, opiniones, temas, aspectos a considerar, etc; organizarlos en grupos según criterios afines y definir, como consecuencia, el aspecto genérico de gestión que identifica a cada grupo (Gómez y González, 1998).

Esta herramienta nos facilita el descubrimiento de conceptos ocultos, ayuda a fortalecer la compresión entre los integrantes del equipo, clarifica ideas y relaciones y descubre ideas nuevas. Las limitaciones y riesgos de su aplicación pueden ser, que no exista una única agrupación posible, que no se debe forzar la información bajo una estructura preconcebida y que algunas ideas pueden no estar asociadas obligatoriamente al resto (Gòmez y Gonzàlez, 1998).

\section{Las fases de desarrollo son:}

Elegir un tópico a estudiar

Recolectar datos/ideas (brainstorming, preguntas sistemáticas)

Escribir datos/ideas sobre tarjetas; una idea por tarjeta

Agrupar las tarjetas en grupos similares Colocar un encabezado sobre cada grupo Revisar cada grupo y añadir nuevas ideas Transferir a un gráfico dibujando una línea alrededor de cada grupo de tarjetas para que se aprecie la delimitación

(Gómez y González, 1998, p. 16).

\section{Diagrama de interrelaciones}

Es también conocida como diagrama de interrelaciones o diagráfico, esta herramienta permite plasmar gráficamente las relaciones existentes entre diversas ideas, temas, problemas, etc; asociada a un tema o problema concreto. Se utiliza tras unos procesos creativos en el que pone de manifiesto que cada idea puede estar relacionada lógicamente con más de una idea. Ello facilita la identificación de todos los aspectos relacionados con un determinado tema $\mathrm{y}$, en su caso, a determinar los diferentes niveles causales (Gómez y González, 1998).

La diferencia entre los diagramas causa-efecto y los diagramas de interrelaciones consiste en que éstos últimos pueden expresar las relaciones más libremente, al no tener que limitarse a un formato particular, aunque esto puede también ser un inconveniente si hay demasiadas relaciones dobles-triples... que hacen difícil establecer un flujo claro de causa-efecto. Además, estos diagramas se adaptan tanto al aspecto operativo como organizativo con lo cual permite identificar las causas principales que afectan de forma grupal (Gómez y González, 1998).

\section{Se consulta de la siguiente manera:}

Se elegirá un tópico a estudiar

Se determinarán las causas, escribiéndolas en tarjetas

Se ordenarán las tarjetas (para un número pequeño se colocarán en círculo; para un gran número se hacen grupos con las que son similares) Dibujar una flecha desde una tarjeta causa a una tarjeta efecto; una causa puede tener varios efectos.

Repetir el paso anterior hasta completar todas las relaciones

Identificar las causas y los efectos clave (la causa clave tiene el número de flechas de salida, el efecto clave tiene el mayor número de flechas de entrada)

Se puede redibujar el diagrama, posicionando la causa clave a la izquierda y le efecto a la derecha, describiendo así un diagrama de flujo (Gómez y González, 1998, pág. 25).

Es de suma utilidad cuando se presentan problemas poco claros que cuentan con causas que se ligan entre sí de forma complicada, y como conclusión ordenada para tener como resultado un diagnóstico detallado (Sotero y Ramírez, 2005).

\section{Diagrama de flujo}

Conocido también como diagrama sistemático o de sistema o diagrama de flujo de sistema, se utiliza para desarrollar gráficamente todas las actividades que deben cubrirse para alcanzar un objetivo dado. El uso de este diagrama permite la identificación de las actividades esenciales, cubriéndolas de forma estructurada y permite subdividir objetivos en actividades realizables. Por otra parte, se convierte en un medio sistemático de referencia para que todas las tareas sean cubiertas y que se den los flujos de trabajo definidos. Así sirve para organizar la información de forma eficiente de lo general a lo específico y genera relaciones causa-efecto multinivel (Gómez y González, 1998).

Un proceso, desde el punto de vista de la organización basada en procesos, consiste en una serie de acciones 
orientadas a generar un valor añadido sobre una entrada, con el fin de obtener un resultado que satisfaga plenamente los requerimientos del cliente, como consecuencia de las actividades realizadas. Todo proceso debe tener un objetivo describible de forma clara (en qué consiste y para qué se realiza). La responsabilidad del mismo ha de quedar asignada a una persona o un equipo concreto que asegure su cumplimiento y eficacia, teniendo bien identificados a sus proveedores y clientes (para quién se realiza). Por último, el resultado final ha de ser mensurable en la cantidad, la calidad y el tiempo invertido en su producción. Para el control de todo proceso es necesaria la creación de indicadores, lo que permite visualizar de forma gráfica su evolución, además de satisfacer la mejora continuada del mismo (planificación, cumplimiento, seguimiento y ajustes para establecer objetivos), (Pinto, Uris y Mena, 2003).

\section{Para construirse este diagrama debe seguirse estos pasos:}

Definir el objetivo final, describiendo claramente todos aquellos aspectos esenciales que puedan interferir

Determinar los principales aspectos o fases a cubrir para alcanzar el objetivo final (evaluando la posibilidad práctica de su realización)

Identificar y priorizar las actividades concretas de cada fase (Gómez y González, 1998, p. 45).

\section{Métodos}

Se emplearon los métodos teóricos como: el histórico lógico, inductivo-deductivo y analítico sintético. Por otra parte, los métodos empíricos que contribuyeron al levantamiento de la información tanto: información primaria como secundaria. El enfoque generado fue cuantitativo por ser la investigación de carácter rígida y estructurada que cumplió con las diferentes fases; entre las más importantes están: el planteamiento del problema, construcción del marco teórico, definición de los objetivos, comprobación de hipótesis y redacción de las conclusiones y recomendaciones del estudio (Del Cid, Méndez y Sandoval, 2011; Hernández, Fernández y Baptista, 2010; Becerra, 2016).

Se partió de un estado exploratorio, debido que estas herramientas de calidad son poco utilizadas y no existe un modelo o estructura en las empresas carroceras, por lo cual fue necesario el entender las diferentes bases teóricas mediante la revisión de la literatura para entender y emprender el problema. Una vez que se comprendió el problema, se direccionó de forma descriptiva con la cual se identificaron las características más importantes de las herramientas de calidad: diagrama de afinidad, de interrelaciones y de flujo. Ya determinadas las características, se encaminó a un estudio correlacional entre las herramientas en mención y las características de la industria metalmecánica, por lo cual fue necesario el establecer dos hipótesis que posterior fueron comprobadas por el modelo estadístico (Coeficiente de Pearson), que entre una de sus cualidades está que facilita entender si existen relaciones positivas o negativas éntrelas variables y sus variables (Fernández, Hernández y Baptista, 2010; Fidias, 2012).

Se empleó la técnica de la encuesta, con su instrumento el cuestionario estructurado, que contó con 23 ítems, de los cuales 3 correspondían a los datos informativos necesarios, 18 de escala de Likert y 2 de opciones múltiples. Cabe mencionar que las preguntas de escala de Likert contaban con dos extremos en un lado "Totalmente en desacuerdo" y el otro "Totalmente de acuerdo" con la opción de neutral en sus respuestas, es decir se tomo el valor 1, 2 y 3. La cual sirvió para la aplicación de la prueba de fiabilidad y aplicación del modelo estadístico (Coeficiente de correlación de Pearson). Los mismos ítems fueron revisados mediante la literatura que se empleó para la construcción del marco teórico.

El instrumento fue aplicado por dos ocasiones, la primera de forma piloto al $20 \%$ de la población en total, es decir, a los gerentes y jefes de producción de las 56 empresas carroceras para conocer si la estructuración del mismo era eficiente $y$, por otro, la consistencia de cada uno de los ítems, en el caso se demostró que era válido el instrumento y se procedió aplicar por segunda vez al total de la población. La población total de empresas carroceras se determinó de acuerdo a la base de datos de la CANFAC, donde constaba el registro de 56 empresas dedicadas a esta actividad perteneciente a la Provincia de Tungurahua, al ser 56 empresas y el instrumento se aplicó a 2 representantes (Gerente y el jefe de calidad en cada empresa) por cada empresa, dando un total de población de 112 personas según el criterio del investigador (Fidias, 2012).

Para la validación del instrumento se realizó en dos partes. La validez que se relaciona si en efecto el instrumento mide lo que se desea, ello se hizo mediante expertos que, para el caso, fueron 4 docentes de la Facultad de Ciencias Administrativas, Carrera de Organización de empresas, quienes en su momento procedieron a dar sus puntos de vista para mejorar el instrumento. Las segunda se refiere a la confiabilidad, que es el "número vez" que es aplicado dicho instrumento en investigaciones pasadas, relacionadas la campo de estudio, lo cual se realizó con 
los datos arrojados en la primera aplicación (piloto), los mismo que se ingresaron al software SPSS Statistics versión 23.0, donde se eligió la opción de fiabilidad (índice de Alfa de Cronbach), pues este índice contribuye en entender si existe consistencia interna entre ítems (Celina y Campo, 2005). El valor arrojado fue de 0,964 que es superior al rango considerado de aceptación que es de 0,70 como indica (Quero, 2010). Ello se puede apreciar en la tabla 1.

Tabla 1. Estadísticas de fiabilidad del instrumento de medición estadística

\begin{tabular}{|c|c|}
\hline Alfa de Cronbach & N. de elementos \\
\hline 0,964 & 23 \\
\hline
\end{tabular}

Fuente: elaboración propia

Finalmente, se definió dos hipótesis: (Ho) Nula: "Las herramientas de calidad no se relacionan con las características económicas del entorno carrocero de la Provincia de Tungurahua" y (Ha) Alterna: "Las herramientas de calidad se relacionan con las características económicas del entorno carrocero de la Provincia de Tungurahua", las mismas que fueron comprobadas con la aplicación del modelo estadístico (Coeficiente de correlación de Pearson), con el cual se determinaron las relaciones positivas y negativas de las herramientas en estudio, con cada una de las características del entono; esto no quiere decir que se buscó la causalidad entre las mismas. Cabe mencionar que el procesamiento de la información y modelo fueron aplicados en el software SPSS Statistics versión 23.0.

\section{Resultados y discusión}

El estudio arrojó datos muy significativos y que repercuten en el desarrollo de las actividades de las empresas carroceras, pues se identificó que la alta gerencia participa activamente en la gestión de la calidad, cumpliendo con las funciones encomendadas a su cargo y direccionando las actividades necesarias para el mejoramiento de las empresas en el aspecto interno y externo (Rivas, 2015).

Constantemente las empresas tratan de evaluar las políticas y planes de calidad, pero es una tarea muy difícil debido a que desconocen formas para hacerlo, es por ello que el seleccionar un sistema de evaluación de la calidad es complicado y va acompañado por ventajas y desventajas, que pueden incurrir demostrado deficiencia en las actividades laborales (Parrilla, Rullán, y Bauzá, 2013).

Por otra parte, los carroceros de la provincia están de acuerdo que debe existir un mejoramiento en los procesos al fabricar sus unidades, pues al existir varios procesos el definir las acciones para dar cumplimiento total a los requerimientos de los clientes es muy difícil, con lo cual se puede incrementar los ingresos y generar más fuentes de empleo en el sector (Cejas, 2013).

El personal encuestado de las carrocerías, comparte que de las herramientas de calidad analizadas la más utilizada en el sector es el diagrama de flujo, porque se pueden graficar las actividades que deben desempeñar en el desarrollo y construcción de las carrocerías, con el cual se puede identificar las actividades esenciales y generales cubriéndolas de una forma estructurada, a fin de lograr los objetivos y disminuir los cuellos de botellas.

Por otra parte, se convierte en un medio sistemático de referencia para que todas las actividades sean cubiertas y que se muestren los flujos de trabajo definidos. Así sirve para organizar la información de forma eficiente de lo general a lo específico y genera relaciones causa-efecto multinivel (Gómez y González, 1998).

Mediante la comprobación de las hipótesis se pudo analizar cada una de las herramientas de estudio para la identificación del impacto con las diferentes características sector carrocero, y cómo ello afecta tanto en lo productivo como la gestión. Para el efecto se empleó el modelo estadístico (Coeficiente de correlación de Pearson), donde se evidenció las relaciones más significativas.

Tabla 2. Correlaciones

\begin{tabular}{|c|c|c|c|}
\hline & & $\begin{array}{c}\text { Comisión de calidad } \\
\text { (departamento) }\end{array}$ & $\begin{array}{c}\text { Reducción } \\
\text { de } \\
\text { materiales }\end{array}$ \\
\hline $\begin{array}{c}\text { Diagrama de } \\
\text { afinidad }\end{array}$ & $\begin{array}{c}\text { Correlación } \\
\text { de Pearson }\end{array}$ & $\mathbf{0 , 9 0 3}^{* *}$ & $0,842^{* *}$ \\
\hline $\begin{array}{c}\text { Diagrama de } \\
\text { interrrelaciones }\end{array}$ & $\begin{array}{c}\text { Correlación } \\
\text { de Pearson }\end{array}$ & $\mathbf{0 , 9 0 3}^{* *}$ & $0,894^{* *}$ \\
\hline $\begin{array}{c}\text { Diagrama de } \\
\text { Flujo }\end{array}$ & $\begin{array}{c}\text { Correlación } \\
\text { de Pearson }\end{array}$ & $0,903^{* *}$ & $\mathbf{0 , 9 2 6 ^ { * * }}$ \\
\hline \multicolumn{2}{|c}{$\mathrm{N}}$. & 112 & $\mathbf{1 1 2}$ \\
\hline
\end{tabular}

**. La correlación es significativa en el nivel 0,01 (bilateral).

Fuente: elaboración propia

Se puede denotar que la correlación de mayor influencia de la primera fila es entre el diagrama de afinidadcomisión con un índice de $(0,903)$, con lo cual se demuestra que existe una correlación positiva alta. Pues el diagrama de afinidad consiste en obtener gran cantidad de ideas, opiniones, temas, aspectos que con una adecuada comisión de calidad en la empresa se puede organizar grupos según criterios afines y establecer, como consecuencia, el aspecto genérico de gestión que identifica a cada grupo para generación de valor en la construcción de cada carrocería (Gómez y González, 1998).

Por otra parte, en la fila dos la correlación de mayor 
aporte fue entre el diagrama de interrelaciones-comisión con un índice de $(0,938)$, con lo cual se demuestra que existe una correlación positiva alta. Pues las empresas no solo requieren identificar ideas o problemas, sino plasmar gráficamente las relaciones existentes con la comisión de calidad asociado a las dificultades y anomalías que se puedan encontrar en los procesos de las empresas. Ello debe estar ligado a los procesos operativos creativos, con lo cual se relaciona las ideas para identificar los diferentes causales del decrecimiento del sector carrocero en la provincia (Gómez y González, 1998).

En la tercera fila se evidencia que la relación de mayor relevancia fue entre los Diagramas de flujo-reducción de materiales, con un índice de $(0,926)$, con lo cual se muestra una correlación positiva alta. Ello debido a que el diagrama de flujo se utiliza para desarrollar gráficamente todas las actividades que deben cubrirse para alcanzar un objetivo dado; si se desarrolla de esa manera es posible reducir la materia prima, además, permite la identificación de las actividades esenciales cubriéndolas de forma estructurada y permite subdividir objetivos en actividades realizables. Por otra parte, se convierte en un medio sistemático de referencia para que todas las tareas sean cubiertas y que se den los flujos de trabajo definidos. Así sirve para organizar la información de forma eficiente, de lo general a lo específico, y genera relaciones causaefecto multinivel involucrando al sector carrocero para mejorar las deficiencias en la calidad de los productos (Gómez y González, 1998).

\section{Conclusiones}

Al revisar la literatura se evidencia que el diagrama de afinidad facilita identificar las diferentes causas, en cuanto a las carrocerías, pues es evidente la mejora que tendrían para poder cristalizar las ideas en los procesos productivos $\mathrm{y}$ organizacionales, generando una mayor utilidad $\mathrm{y}$ desafíos para el crecimiento económico del sector.

El diagrama de interrelaciones es una buena herramienta para identificar ideas o problemas que se plasmaran gráficamente las relaciones existentes entre los diferentes departamentos y procesos de producción, a fin de poder identificar las semejanzas entre las actividades y tener una variable en concreto que se pueda generar un valor agregado para la generación de políticas de mejora en la fabricación de carrocerías.

Luego de revisar las diferentes herramientas de calidad se puede concluir que la herramienta más utilizada en el sector car rocero es el diagrama de flujo, porque esta permite plasmar en forma gráfica las actividades a desempeñar en el desarrollo y construcción de las carrocerías, además, facilita la identificación de las actividades esenciales y generales, cubriéndolas de una forma estructurada para lograr los objetivos e identificar los cuellos de botellas que, en gran medida, son los causantes del decrecimiento económico del sector. Por otra parte, se convierte en un medio sistemático de referencia para que todas las actividades sean cubiertas y que se den los flujos de trabajo definidos para el crecimiento de las empresas.

\section{Referencias}

1. Becerra, G. (2016). Los usos del constructivismo en las publicaciones científicas de Latinoamérica. Revista Mad. Revista del Magíster en Análisis Sistémico Aplicado a la Sociedad (35), 38-59. DOI: 10.5354/0718-0527.2016.42796.

2. Bernal, H., y Mungray, A. (2017). Los índices de competitividad en México. Gestión y Política Pública, 26(1), 167-218.

3. Bombón, D., Vayas, G., Villacis, J., y Gutierrez, E. (2017). Caracterización del entorno empresarial proveedor del sector carrocero de la provincia de Tungurahua. Congreso internacional Ciencia Sociedad e Investigación Universitaria. Ambato.

4. Cejas, C. (2013). Mejoras en la gestión del proceso editorial. Revista Argentina de Radiología, 77(3), 193-194.

5. Parrilla, J. C., Rullán, X. O., y Bauzà, M. S. (2013). Evaluando la evaluación continua. @ tic. revista d'innovació educativa, 10, 33-43.

6. Celina, H., y Campo, A. (2005). Aproximación al uso del coeficiente alfa de Cronbach. Colombiana de Psiquiatría, 34(4), 572-580.

7. Del Cid, A., Méndez, R., y Sandoval, F. (2011). Investigación. Fundamentos y metodología ( $2^{\circ}$ ed.). Nauclapan de Juárez, México: Pearson Educación.

8. Fernández, C., Hernández, R., y Baptista, M. (2010). Metodología de la Investigación (Quinta ed.). Distrito Federal, México: Mc Graw-Hill. ISBN: 978-607-15-0291-9.

9. Fiallos, J. G. (2014). Estudio de la cadena productiva del sector metalmecánico -carrocero de la provincia de Tungurahua cantón Ambato. Universidad Central del Ecuador. Facultad de Ciencias Económicas. Escuela de Economía. Tesis de pregrado. Quito. Obtenido de: http://www.dspace.uce.edu.ec:8080/ 


\section{bitstream/25000/3044/1/T-UCE-0005-461.pdf}

10. Fidias, G. (2012). El proyecto de investigación $\left(6^{\circ}\right.$ ed.). Caracas, Venezuela: Episteme. ISBN: 980-078529-9.

11. Gándara, F. (2014). Herramientas de calidad y el trabajo en equipo para disminuir la reprobaciòn escolar. Conciencia Tecnológica (48), 17-24.

12. Gómez, B., y González, Y. (1998). Gestión estratégica de la calidad. Herramientas: una aplicación en el campo sanitario. Anales de estudios económicos y empresariales (13), 275-316.

13. Heras, I., Marimon, F., \& Casadesús, M. (2009). Impacto competitivo de las herramientas para la gestión de la calidad. Cuadernos de Economía y Dirección de la Empresa, 12(41), 7-35.

14. Pérez, M. (2017). Implementación de herramientas de control de calidad en MYPEs de confecciones y aplicación de mejora contínua PHRA. Industrial Data, 20(2), 95-100.

15. Pinto, C., Uris, J., y Mena, L. (2003). El diagrama de flujo, herramienta para la gestión de procesos en una Unidad de Admisión hospitalaria. Papeles Médico, XII(3), 119-124. Obtenido de http://sedom.es/wp-content/themes/sedom/ pdf/4cbc747cda70apm-12-3-005.pdf.
16. Quero, M. (2010). Confiabilidad y coeficiente Alpha de Cronbach. Telos, XII(2), 248-252. ISSN: 1317-0570.

17. Rivas, L. (2015). El equipo de alta gerencia en una empresa multinegocios. Caso suramericana S.A. Ciencias Estratégicas, XXIII (23), 121-133.

18. Sotero, O., y Ramírez, L. (2005). Del método científico al diagnóstico de problemas de ingeniería en México. Ingeniería Investigación y Tecnología, II, 139-146. Obtenido de http://www.scielo.org.mx/ pdf/iit/v6n2/1405-7743-iit-6-02-139.pdf.

19. Talavera, C. (2013). Métodos y Herramientas de Mejora aplicados en la Administración Pública. Unión Iberoamericana de Municipalistas. ISBN: 978-84-937777-6-0.

20. Viega, A., Quintero, J., y Romero, L. (2017). Diagrama de afinidad (Método KJ) para la construcción curricular. Revista Equidad, 1, 113119. 